NBER WORKING PAPER SERIES

\title{
OPTIMAL FUNDING AND ASSET ALLOCATION RULES \\ FOR DEFINED-BENEFIT PENSION PLANS
}

\author{
J. Michael Harrison \\ William F. Sharpe
}

Working Paper No. $\underline{935}$

\section{NATIONAL BURFAU OF ECONOMIC RESEARCH 1050 Massachusetts Avenue Cambridge MA 02138}

$$
\text { July } 1982
$$

Paper presented at the National Bureau of Economic Research Conference on Financial Aspects of the U.S. Pension System, March 1982. The research reported here is part of the NBER's research program in Pensions. Any opinions expressed are those of the authors and not those of the National Bureau of Fconomic Research. 
NBER Working Paper \#935

July 1982

Optimal Funding and Asset Allocation Rules for
Defined-Benefit Pension Plans

\section{ABSTRACT}

This paper considers a world in which pension funds may default, the cost of the associated risk of default is not borne fully by the sponsoring corporation, and there are differential tax effects. The focus is on ways in which the wealth of the shareholders of a corporation sponsoring a pension plan might be increased if the Internal Revenue Service (IRS) and the Pension Benefit Guaranty Corporation (PBGC) follow simple and naive policies. Under the conditions examined, the optimal policy for pension plan funding and asset allocation is shown to be extremal in a certain sense. This suggests that the IRS and the PBGC may wish to use more complex regulatory procedures than those considered in the paper.

J. Michael Harrison William F. Sharpe Graduate School of Business Stanford University Stanford, CA 94305

(415) 497-4492 


\section{Introduction}

Considerable attention has been devoted to the funding of defined-benefit pension plans. Both the level of funding and the allocation of fund assets have been considered from the viewpoints of various interested parties beneficiaries, corporate managers, corporate shareholders, and the Pension Benefit Guaranty Corporation). Eoth practical and theoretical investigations have tended to characterize the asset allocation decision as one of choosing an appropriate "bond-stock mix".

Sharpe (1976) showed that, in the absence of taxes, if the parties bearing the, cost of possible default behave rationally, neither the asset allocation decision nor the funding decision may affect the wealth of corporate shareholders. On the other hand, if some parties do not require compensation for actions that increase the risk of default, the optimal policy from the viewpoint of corporate shareholders may involve funding as little as possible and using asset allocation to maximize default risk.

Two important papers, by $B l a c k$ (1980) and Tepper (1981) consider the effects of current tax law, assuming that there is no probability of default! Under these conditions shareholder wealth may be maximized by funding to the greatest possible extent and holding assets (such as bonds) taxed highly for other investors.

This paper considers a world in which pension funds may default, the cost of the associated risk of default is not borne fully by the sponsoring corporation, and there are differential tax effects. We explore ways in which the wealth of the shareholders of a corporation sponsoring a pension plan might be increased if the Internal Revenue Service (IfiS) and the Fension Benefit Guaranty Corporation (PBGC) follow simple (and naive) policies. This analysis suggests that the two agencies may want to consider more complex rules. Optimal policy 
Funding and Allocation Fules for Fension Flans

for the FEGC is discussed briefly in Sharpe (1976); Tepper (1982) describes some of the issues involved in setting tax policy. We do not explore such issues here. Instead, we hope to provide useful inputs for addressing these more fundamental questions. 
2. The General Froblem

Throughout, we will deal with a defined benefit plan. An initial decision must be made concerning the amount of assets in the plan. Following this decision, we assume that the plan will be frozen. That is, no further benefits will accrue and no further contributions will be made.

In addition to the decision concerning the level of initial. assets, decisions must be made in subsequent periods concerning the allocation of assets among alternative investment instruments. The former is termed the funding decision, the latter are asset allocation decisions. All may be constrained by legal, regulatory, or other restrictions..

The liabilities of a frozen defined-benefit plan can be described by a vector of benefit payments $B_{1}, B_{2}, \ldots . E_{T}$ to be paid from the fund at times 1,2,..T if possible. We assume these are known with certainty. 2

At each time $t$, the current market value of the fund's assets is compared with the benefits then due. If assets exceed required payments, the benefits are paid and the remaining assets re-invested. If not, no further benefits are paid by the fund. If all benefits have been paid at date $T$, the remaining assets revert to the sponsoring corporation.

Eeneficiaries may be insured in whole or in part against default, but we assume that neither insurance premia nor wages paid by the corporation are affected by the level of funding or the allocation of fund assets. Moreover, we assume that the goal of those making these decisions is to maximize the wealth of corporate shareholders." 


\section{1 Vallation of Contingent Claims}

To represent a market with "tax effects" we employ a modified version of the statempreference approach used by (Litzenberger and Vantorne , 1978). Individual investors can purchase claims to receive income contingent on the occurrence of alternative states of the world. However, different binds of payments may be taxed differently for at least some investors. Thus a claim for a "rapital gain dollar" contingent on state $s$ may be valued differently in the market than one for a "dividend dollar" contingent on the same state. Given M such types of dollars and $N$ states of the world, we assume that it is possible, explicitly or implicitly, for individual investors to purchase all M*N primitive (Arrow-Debreu) contingent claims. This is similar, buţ not the same as, the familiar complete-markets approach. The latter typically assumes that existing securities "span" the space of state-contingent claims and that unlimited short sales with no impounds and full use of the proceeds are possible. In a taxable world, if this were possible, riskless "tax arbitrage" between two investors in different tax brackets could occur, as shown by lschaefer, 1979). Thus we implicitly assume the existence of boundaries on holdings andfor tax treatment.

We assume that at time $T$, the residual walue of the fund, R (which will be zero if the fund has defaulted) will be lused to pay shareholders. The amount may be subject to corporate tax leaving less than $F$ to be paid out. Let

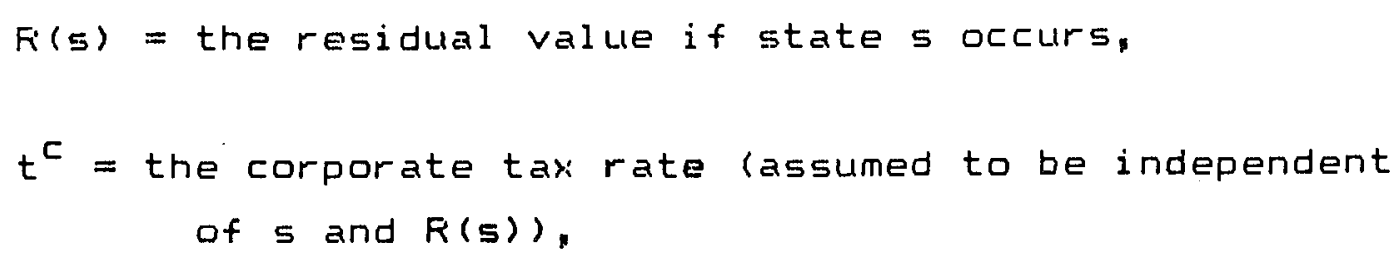

$$
\begin{aligned}
v^{a}(s)= & \text { the present value of a dollar paid to } \\
& \text { the corporation's shareholders in state } \mathbf{s} .
\end{aligned}
$$




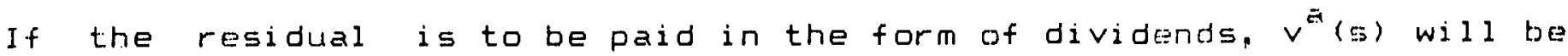
the marlet price for a "dividend dollar" in state 5 ; if the residual is to be paid in capital gains, $v^{a}(s)$ will be the market price of a "Capital gains dollar" in state 5 . We assume only that the form each distribution would take is known, and that the $v^{2}(s)$ values are selected accordingly.

Letting:

$$
v(s)=v^{a}(s)\left[1-t^{c}\right]
$$

the present value of $F(s)$ is

$$
V(s)=V(s) F(s) .
$$

Henceforth we will focus on the $v(s)$ values, with $v(1), \ldots, v(N)$ termed the baluation function.

Given a set of possible residual values $F(1), \ldots, F(N)$, the value of the pension fund for the corporate shareholders will be

$$
V=E_{:}^{*} V(s)=\bar{\Xi}_{5}[V(s) F(s)]
$$

We assume that the objective of those making the decisions concerning the fund is to maximize $V$, given some valuation function $v(1), \ldots, V(N)$.

A bey ingredient in our analysis is the assumption that neither the corporate taxes nor the personal taxes paid on $F(s)$ will be related to the choice of investments made by the managers of the pension fund. For example, the eventual taxes paid if a "dividend dollar" is received within the pension fund in a given state of the world would be the same as those paid if a "capital gains" dollar had been received within the fund in the same circumstances. A dollar received in the pension fund in a given state of the world thus has the same value for the corporation's shareholders, whatever its type. However, the present price of a claim to receive such a dollar may depend on its type. Thus the presence of differential tax treatment 
will influence the relative attractiveness of alternative instruments, even for a "tax-exempt" pension fund.

\subsection{Choice of Assets}

While individuals may have some flexibility in their choice of state-contingent payments, we assume that the pension fund must choose from a limited set of combinations of such clains. The fund manager"s choice set thus does not span the set of state-contingent claims. In the next part of the paper we consider a choice between two instruments ( e.g., "bonds" and "stocks") in a one-period setting. We show that in this case one of two extreme funding policies (fund either as much or as little as possible) will be optimal, as will one of two extreme asset allocation policies ( invest either in bonds or in stacks, but not both). Later we obtain analogous results in a setting involving many periods and many assets. We then address the issue raised by Elack and Tepper: given two instruments, bonds and stocks, with different tax status and different risks, we show that an all-bond, maximum-funding policy may not be optimal. The final section of the paper provides suggestions for future research. 
We begin with the asset allocation decision.

Consider a pension plan with A dollars to invest. This may be used to purchase either or both of two types of assets. One unit of asset 1 can be purchased for one dollar and will pay $D^{1}(s)$ dollars one period hence if the state of the world is s. Similarly, one unit of asset 2 can be purchased for one dollar and will pay $D^{2}(s)$ dollars in one period if state $s$ occurs. We assume a simple regulatory setting in which the plan's managers may select a value of $x$ between $x_{m i n}$ and

$x_{\text {max: }}$ where

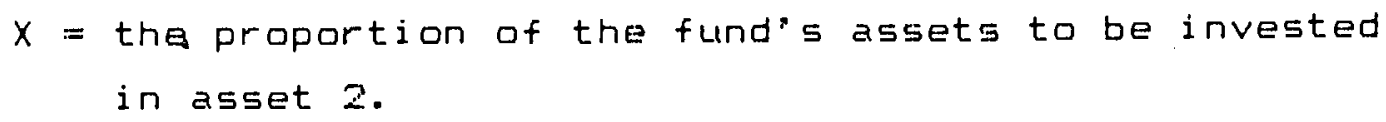

We also assume that there are no transactions costs.

The value of assets one period hence will be

$$
(1-X) A D^{1}(s)+X A D^{2}(s)
$$

At the end of the period, a benefit of $E$ dollars must be paid out of the fund's assets, if possible. The amount (if any) left for taxes and payments to shareholders will be

$$
F(A, X, s)=\left[(1-X) A D^{1}(s)+X A D^{2}(s)-B\right]^{+},
$$

where

$$
[z]^{+} \text {denotes } \Sigma \text { if } z>0 \text { and } 0 \text { if } z<0 \text {. }
$$

Assume that neither the firm's wage bill nor its insurance premium is a function of $X$ or $A$. 
The amount of the residual claim in state $s$ is

$$
R(A, X, s)=\left\{A\left[D^{1}(s)+X\left(D^{2}(5)-D^{1}(s)\right)\right]-E\right)^{+}
$$

The goal is to maximize the value of the residual claim

$$
V=\overline{\frac{F}{5}} \quad V(s) F(A, x, 5)
$$

by choosing feasible values of $A$ and $X$.

Let

$$
\begin{aligned}
V(s) & =V(5) F(A, X, 5) \\
& \left.=V(5) \& A\left[D^{1}(5)+X\left(D^{2}(5)-D^{1}(5)\right)\right]-B\right)^{+}
\end{aligned}
$$

Figure 1 plots $V(s)$ as a function of $x$, given $A$, for three cases: (a) a state in which asset 2 underperforms asset 1 , (b) a state in which the two assets have equal payoffs, and (c) a state in which asset 2 outperforms asset 1 . A key observation is that the function is (weatily) convex from above -- going from left to right the sjope never decreases.

FIGURE 1 HERE

Note that $V$ is simply the sum over states of such components of value:

$$
V=\frac{1}{5} V(s)
$$

Thus it must also be weakly convex from above.

It follows that, given $A$, shareholder wealth will be maximized at either $x_{m i n}$ or $x_{m a x}$ but every interior feasible value of $x$ will be dominated. Depending on the situation, all funds should be invested in either asset 1 or asset 2. No mixed solutions will be optimal.

Now consider the question of the appropriate funding level. Assets invested in a pension fund must come from somewhere. corporate 
projects must be foregone, funds must be raised from bondholders or stockholders, etc. For simplicity, assume that dollars not invested in the fund will be leed to finance an investment that will pay $D^{C}(s)$ dollars one period hence if the state of the world is 5 and that suich payoffs will be tayed in the same manner as those obtained as residual values from the pension fund. Then the opportunity cost of an investment of $A$ dollars in the fund will be

$$
A \underset{5}{S}\left[V(5) D^{C}(5)\right]=A V^{C} \text {, }
$$

where

$$
\begin{gathered}
V^{C}=\text { the present value of } 1 \text { dollar invested in corporate } \\
\text { assets (at the margin, } 1 \text { dollar), }
\end{gathered}
$$

and the net present value of the pension fund to the shareholders will be

$$
v^{n}=\operatorname{sUM}_{s}\left[V(s)-V(s) D^{[}(s)\right] .
$$

Equivalently stated:

$$
v^{n}=\bar{s} \cdot v^{n}(s)
$$

where

$$
\begin{aligned}
& V^{n}(s)=V(s)\left\{[A r(s)-E]^{+}-A D^{C}(s)\right\} . \\
& r(s)=(1-X) D^{1}(s)+X D^{2}(s) .
\end{aligned}
$$

The value of $v^{n}$ will typically be negative; thus ( $-v^{n}$ ) can be interpreted as the cost of the pension plan. We assume that the goal is to maximize the former value -- i.e. to minimize the cost of the plan.

Figure 2 shows the assumed regulatory climate. The fund may choose a value of $A$ between $A_{m i n}$ and $A_{\text {max }}$ and a value of $x$ between $x_{m i n}$ and $x_{\max }$. The value of $A_{\max }$ might correspond to ERISA's full funding 
Funding and Aldocation Rules for Pension Plang

Page 11

limitation and $A_{m i n}$ to its minimum funding standard. The valueg of $x_{\min }$ and $x_{\text {max }}$ are iritended to represent a naive policy on the part of the FEGC concerning "prudent" management of the fund. In this simple case the feasible region has four "corners". In section 4 we consider a somewhat : broader class of regulatory policies; however, the assumption is retained that the feasible region is convex, with linear borders.

\section{FIGUFE ? HERE}

Figure $\Xi$ plots $V^{n}(s)$ as a function of $A$, given $X$, for three cases: (a) a state in which pension assets do better than corporate assets, (b) atate in which the two types of assets do equally well, and (c:) a state in which pension assets underperform corporate assets. This function is also (weakly) convex from above. And, since $v^{n}$ is the sum over states of such components of value, it must also be weatily conver, from above.

FIGURE 3 HERE

It follows that, given $x$, shareholder wealth will be maximized at. either $A_{m i n}$ or $A_{m a x}$ but every interior feasible walue of a will te dominated. Depending on the situation, one should fund a plan as much or as little as possible.

Given A, an extreme value of $X$ should be chosen. Given $X$, an extremea value of $A$ should be chosen. The overall optimum thus involves an extreme value of $A$ and an extreme value of $X$. In Figure 2 , all positions but those at the corners of the feasible region will be dominated. 
4. Many Feriods and Many Assets

Consider now a more general problem with defined benefits $B_{1}, \ldots . B_{T}$ payable at times 1,..,T. Further suppose that the pension plan can invest in $r$ assets, indexed by $k=1, \ldots, k$. Generalizing the notation used earlier, we denote by $D_{t}^{k}$ the return at time $t$ to a dollar invested at $t i m e t-1$ in security $k$. of course $D_{t}^{k}$ further depends on the state $s$, but it will be convenient to suppress this dependence initially.

Next let $x_{t}^{k}$ denote the fraction of the pension plan's wealth that is invested in security $t$ between $t$ imes $t-1$ and $t$, and define

$$
\text { (1) } M_{t}=\sum_{t=1, k} \quad x_{t} D_{t}^{k} k \text { for } t=1, \ldots, T
$$

If a total of $z$ dollars is invested at time $t-1$; the wealth of the pension fund at $t i m e$, before payment of benefits, will be $Z M_{t}$. We call Mt the pension fund"s investment multiplier for period t. The portfolio proportions $x_{t}^{k}$ are decision variables and can depend on the state $s$ through information that is available at time $t-1$ (see below), but. we surpress this dependence for the moment. The pension fund manager's other decision variable is the initial investment level $A$. We denote by $w_{t}$ the wealth of the pension fund at $t i m e t$, immediately after payment of benefits. These wealth levels $W_{t}$ can be expressed in terms of the initial investment level $A$ and portfolio proportions $x_{t}^{k}$ by the recursive formula

(2) $w_{t}=\left(w_{t-1} M_{t}-E_{t}\right)^{+}$for $t=1, \ldots, T$

with $W_{0}=A$ by convention.

(3) Proposition. The residual wealth $R=W_{T}$ is a convex function of each multiplier $M_{t}$ alone, holding the other multipliers, the benefit. obligations, and the initial investment level fixed. Furthermore, $R$ is a convex function of the initial investment level A, holding all else fixed. 
proof. Let $t$ be arbitrary, let $M_{t}=0$ and $M_{t}^{\prime \prime}=0$ be two possible values for the multiplier in period $t$, and set $M_{t}=.5 M_{t}^{*}+5 M_{t}^{\prime \prime}$ Let $W_{1}, \ldots, W_{T}$ be defined by (2), with $M_{t}^{\prime}$ in place of $M_{t}$ (plus the convention $W_{0}^{\prime}=A$ ), and let $w_{0}^{\prime \prime} w_{1}^{\prime \prime}, \ldots, w_{T}^{\prime \prime}$ be defined similarly. To prove the first statement. of the proposition, we need to show that $w_{T}=.5 w_{T}^{+} .5 w_{T}^{\prime \prime}$ obviously $w_{i}=w_{i}=w_{i}^{\prime \prime}$ for $i=0, \ldots, t-1$. Then

$$
\text { (4) } \begin{aligned}
w_{t} & =\left(W_{t-1} M_{t}-E_{t}\right)^{+}=\left[W_{t}\left(.5 M_{t}^{\prime}+.5 M_{t}^{\prime \prime}\right)-B_{t}\right]^{+} \\
& =\left[.5\left(W_{t-1} M_{t}^{\prime}-E_{t}\right)+.5\left(W_{t-1} M_{t}^{\prime \prime}-E_{t}\right)\right]^{+} \\
& =.5\left(W_{t-1} M_{t}^{\prime}-E_{t}\right)^{+}+.5\left(W_{t-1} M_{t}^{\prime \prime}-E_{t}\right)^{+} \\
& =.5 W_{t}^{\prime}+.5 W_{t}^{\prime \prime} .
\end{aligned}
$$

because (.) $)^{+}$is a convex function. Eut (.) ${ }^{+}$is also an increasing function, so from (4) we have next that

(5)

$$
\begin{aligned}
w_{t+1} & =\left(w_{t} M_{t+1}-E_{t+1}\right)^{+} \\
< & =\left[\left(.5 w_{t}^{\prime}+.5 w_{t}^{\prime \prime}\right) M_{t+1}-B_{t+1}\right]^{+} \\
& =\left[.5\left(w_{t}^{\prime} M_{t+1}-E_{t+1}\right)+.5\left(w_{t}^{\prime \prime} M_{t+1}-B_{t+1}\right)\right]^{+} .
\end{aligned}
$$

Using again the convexity of $(.)^{+}$,

(6)

$$
\begin{aligned}
{\left[.5\left(w_{t}^{\prime} M_{t+1}-B_{t+1}\right)+.5\left(w_{t}^{\prime \prime} M_{t+1}-E_{t+1}\right)\right]^{+} } \\
<=.5\left(w_{t}^{\prime} M_{t+1}-B_{t+1}\right)^{+}+.5\left(w_{t}^{\prime \prime} M_{t+1}-B_{t+1}\right)^{+} \\
=.5 w_{t+1}^{\prime}+.5 w_{t+1}^{\prime \prime} .
\end{aligned}
$$

Conmbining (5) and (6) gives $w_{t+1}<=.5 w_{t+1}^{\prime}+.5 w_{t+1}^{\prime \prime}$ and one can obviously continue in this way to prove by induction that $W_{T}{ }^{\prime}=.5 W_{T}^{*} .5 W_{T}^{\prime \prime}$. This completes the proof of the first statement.

To prove that $W_{T}$ is a convex function of the initial investment level, 
let $A^{\prime} Y=0$ and $A^{\prime \prime} y=0$ be arbitrary, and set $A=.5 A^{\prime}+.5 A^{\prime \prime}$. Set $W_{0}^{\prime}=A^{\prime}$ and $w_{0}^{\prime \prime}=A^{\prime \prime}$, and define $W_{t}, w_{t}^{\prime}$ and $w_{t}^{\prime \prime}$ in the obvious way for $t=1, \ldots, T$. We need to show that $W_{T}<=.5 W_{T}^{+} .5 W_{T}^{\prime \prime}$. Steps (5) and (6) above can be lised inductively to prove the stronger result that $w_{t} s=.5 w_{t}+.5 w_{t}^{\prime \prime}$ for aII $t=1, \ldots, T$. Thus the proof of the proposition is complete.

\subsection{Formulation of the Fund Manager"s Froblem}

We assume that the pension fund manager wants to select an initial funding level $A$ and relative portfolio proportions $x_{t}^{k}$ so as to maximize the valuation of the residual claim $F=W_{T}$ subject to regulatory limitations imposed on the initial funding level or fund allocation among assets. One must also specify the fund manager's capability to adjust the allocation dynamically in response to information received. The latter aspect malies formal representation of multi-period: problems fundamentally more complex than single-period problems. We will adopt a rather abstract representation of the manager"s optimization problem, but one that is well suited to our ob jectives.

Let $\therefore$ be the set of all states $s$ that might pertain at time T. A trading strategy will be formally defined as a collection $\left(A, x_{1}, \ldots, x_{T}\right)$ where

(i) A is a constant,

(ii) $x_{1}=\left(x_{1}^{1}, \ldots, x_{1}^{k}\right)$ is a vector whose components sum to one, and for $t=2, \ldots . T$

(i.ii) $x_{t}=\left(x_{t}^{1}(s), \ldots, x_{t}^{k}(s)\right)$ is a vector of functions whose components sum to one for every state $s$

One interprets $x_{t}^{k}(s)$ as the fraction of the fund"s wealth to be invested in security $k$ during period $t$ if state $s$ prevails, and it is obviously necessary to restrict the way in which this fraction may depend on $s$. We tale as given a sequence $F_{1}, \ldots . F_{T-1}$ of successively 
finer partitions of as with cells of the partition f representing those events whose occurrence or non-occurrence will be bnown at time $t$. In addition to the restrictions stated above, it is required that $x_{t}$ (viewed as a vector-valued function of $s$ ) be measurable with respect to $F_{t-1}$, meaning that $x_{t}(s)=x_{t}\left(s^{*}\right)$ whenever $s$ and $s^{\prime}$ lie in the same cell of the partition $F_{t-1}$. Ey defining $F_{0}$ as the trivial partition (whose only cell is a itself), we can extend this measurability requirement to apply to $x_{1}$ as well. Eecause $a$ is a finite set. by assumption, each of the strategy components $x_{t}^{k}$ can be viewed as a finite-dimensional vector rather than a function, this vector having one component for each cell of the partition $F_{t-1}$.

To complete the formulation, we require that $A=I$ and that $x_{t}(s)=2$ for all $t=1, \ldots T$ and $5 \mathrm{~T}$, where $I$ is a compact interval and $\Delta$ is a set to be described shortly. As in the model of section 2, I is the interval between a lower funding limit imposed by the FEGC and an upper limit imposed by the IF's, while 4 is the set of all asset distributions (relative portfolio proportions) that are judged by the figc to be prudent. For the two-asset model of section 2 we took is to be the set of all pairs $\left(x^{1}, x^{2}\right)$ such that

$$
x_{\min }<=x^{1}=x_{\max } \quad \text { and } \quad x^{1}+x^{2}=1
$$

For the gerieral setting we assume that $\&$ is the bounded solution set of some finite system of linear inequalities and equalities, including the requirement $x^{1}+\ldots+x^{k}=1$. (Thus i is a compact, convex, polyhedral set and has a finite number of extreme points.)

The preceding paragraph contains a strong assumption. We are assuming that the definition of a prudent asset distribution does not depend on the initial funding level $A$, and more generally does not depend on the success enjoyed by the fund"s investments up to an intermediate decision point. This assumption is essential in all that follows, although $A$ can be allowed to depend on $t$ and even $s$ (subject to measurability restrictions) without substantially changing our analysj. 5.

For $t=1, \ldots, T$ let $\Gamma_{t}$ be the set of all functions $x_{t}$ that map $a$ into $A$ 
and are neasurable with respect to $F_{t-1}$. The preceding discussicin may te summarized as follows. The pension fund manacger must choose AEI, $x_{1}=\Gamma_{1}, \ldots, x_{T} r_{T} 50$ as to maximize

(7) $\quad V=\frac{V}{5}: V(5)\left[F(5)-A D^{C}(5)\right]$.

where $v($.$) is the valuation function discussed in section 2$, and $R(s)$ is defined in terms of $A$ and the relative portfolio proportions $x_{t}^{t}$ (s) as at the beginning of this section.

Ours is not a standard dynamic programming formulation of the fund manager"s sequential decision problem. In particular. we have mede no explicit mention of the way current portfolio decisions may depend on the fund wealth carried forward from previous periods. By coordinating the way successive portfolio descriptions $x_{1}, \ldots . x_{T}$ are made to depend on $s$, however, one can synthesize any desired dependency of current decisions on past success or failure. Our formulation is completely equivalent to the standard one. For future reference, we observe that this problem does have an optimal solution (the supremum is attained). because it amounts to maximization of a continuous function over a compact subset of a finite-dimensional Euclidean space.

\subsection{Optimality of Extremal Strategies}

Hereafter we denote by $x=\left(x^{1}, \ldots, x^{k}\right)$ a generic element of $a$, by $y=\left(y^{1}, \ldots . y^{k}\right)$ a generic extreme point of $\Delta$, and by $E$ the (finite) set. of all such extreme points y. Since i is a compact, convex, polyhedral set by assumption, each point $x$ s can be written as a convex combination of the extreme points $y$. That is, there exist non-negative weights $[a(y), y=E]$ such that $\bar{y} a(y)=1$ and

(8) $\quad x=\overline{\bar{y}} a(y) \quad y$;

or equivalently.

(9) $x^{k}=F_{y} \quad a(y) y^{k} \quad$ for $k=1, \ldots, k$ 
A strategy $(A, X \quad 1 . . . X$ f for the fund manager's optimization problem is said to be extremal if $A$ is an endpoint (extreme point) of the interval I and $x_{t}(s)=E$ for each state $s$ and each $t=1, \ldots, T$. Incidentally: a single strategy component $x_{t}$ will be called extremal if $x_{t}(s): E$ for all states 5 .

It is the purpose of this subsection to prove there exists an optimex strategy which is extremal. Toward that end, first 1 et $\left(A, x_{1}, \ldots, x_{7}\right)$ be an arbitrary feasible strategy. Adding a notational dependence on $A$ for emphasis, let $F(A, 5)=W_{T}(A, s)$ be defined in terms of $A$ and the relative portfolio proportions $x_{t}^{k}(s)$ as at the beginning of this section, and then let $V(A)$ be defined in terms of $R(A, 5)$ by (7). Froposjtion (S) shows that $F(A, S)$ is a conver function of $A$ for each fixed 5 , and it follows immediately (because the contingent claim valuations $V(s)$ are non-negative) that $V(A)$ is convex. Thus $V(A)$ will be maximized by taking $A$ to be an endpoint of the feasible interval I. To repeat, the valuation associated with an arbitrary feasible strategy can be increased (or at least not decreased) by moving $A$ to one of the extreme points of $I$, leaving $x_{1} . . . . x_{T}$ fixed.

We now argue that a similar improvement can be effected by substituting for any of the strategy components $x_{t}$ a well chosen extremal strategy component. Again let $\left(A, x_{1}, \ldots, x_{T}\right)$ be arbitrary, $f i x$ a period $t$, and let $S$ be any cell of the partition $F_{t-1}$. Then there exists a point $x \in$ such that $x_{t}(s)=x$ for all ses. Adding a notational dependence on $\because$ for emphasis, let us set

$$
\begin{aligned}
(10) M_{t}(s, x) & =E_{k=1, k} x_{t}^{k}(s) D_{t}^{k}(s) \\
& =I_{k=1, k} x^{k} D_{t}^{k}(s) \text { for } s s .
\end{aligned}
$$

Then $R(5, x)=W_{T}(5, x)$ is defined in terms of $M_{t}(5, x)$ for states 5 es as at the beginning of this section, and we set

$$
V(S, x)=E_{5 S S} V(5)\left[F(5, x)-A D^{C}(5)\right] .
$$

Obviously $V(S, x)$ is the contribution to the total valuation $V$ made by 
states in cell $\mathrm{S}$, and it is only the contribution from these states that is affected by our choice of $x$.

Fiecall that $x$ can be represented in terms of the extremal points $y v i a$ (8), and we now consider how the partial valuation $v(5)$ would be affected if we were to replace $x$ by some yeE. By analogy with (10), let

(11) $M_{t}(s, y)=I_{t:=1, k} y D_{t}^{k}(\stackrel{k}{s})$ for $y=E$ and $s: s$,

and then let $F(s, y)$ and $V(S, y)$ be defined in terms of $M_{t}(s, y)$ in the obvious way. From (8), (10) and (11), we have

$$
M_{t}(s, x)=E_{y E} \text { a }(y) M_{t}(s, y) \text { for } s: s
$$

Froposition (3) says that $k=W_{T}$ is a convex function of $M_{t}$ " 50 (12) implies

(13) $F(5, x)<=\quad F_{y \in E} a(y) F(5, y)$ for $5: 5$,

and hence (because $v(5) y=0$ for all $5 s s$ )

(14) $\quad V(S, x)<=\quad E_{y \leq S} a(y) \quad V(S, y)$.

obviously (14) can only hold if $V(S, y) y=V(5, x)$ for at least one extreme point $y$, so we can increase (or at least not decrease) $V(s)$ by substituting this $y$ for $x$ in our specification of the strategy component $x_{t}$. Fiepeating this argument for each cell of the partition $F_{t-1}$, we come to the following: one can increase the overall valuation $v=\frac{5}{5} v(s)$, or at least not decrease it, by substituting for $x_{t} a n$ extremal strategy component $x_{t}^{*}$.

The proof that there exists an extremal optimal policy is now essentially complete. We know that there exists an optimal solution $\left(A, x_{1}, \ldots, x_{T}\right)$. But one can substitute for $A$ an extremal investment level $A *$, then substitute for $x_{1}$ a well chosen extremal strategy component $x_{1}^{*}, \ldots$, then substitute for $x_{T}$ a well chosen extremal strategy component $x_{T}^{*}$, without ever decreasing the total valuation. 
Funding and Allocation Fules for Fension Plans Fage 19

fbsolutely essential to this argument is the assumption that one can take $A$ to be any point in the interval $I$, and $x_{t}$ to be any element of ' $t$, regardiess of how the other components of the overall strateqy have been selected. 


\section{Elonds Versus Stockis}

We turn now to the issues raised by Elack (1980) and Tepper (1981). Given a choice between "bonds" and "stoct:s" and a range of permissible funding levels, what poljcies will be optimal for corporate shareholders? To analyze these issues we will use the simple one-period, two-asset setting of section 5 .

Key to the Black-Tepper argument is the superiority of pension fund investment over corporate investment and, within the pension fund, the superiority of bonds (here, asset 1) over stocks (asset 2). In our notation this assumption tates the form:

(15) $\quad \frac{5}{5} \quad V(5) D^{1}(5)>\quad V(5) D^{2}(5) \geqslant \overline{5} v(5) D^{C}(5)$

From (15) it is easy to deduce the following: if all feasible $(A, X)$ combinations provide adequate coverage to pay every beneficiary in full in every state of the world, the optimal solution will involve full funding ( $\left.A=A_{\text {max: }}\right)$ and investment solely in bonds $(X=0)$. The argument goes as follows. Since there will be no default in any state, the positive part $\left([.]^{+}\right)$notation becomes redundant and the value of the residual can be written as:

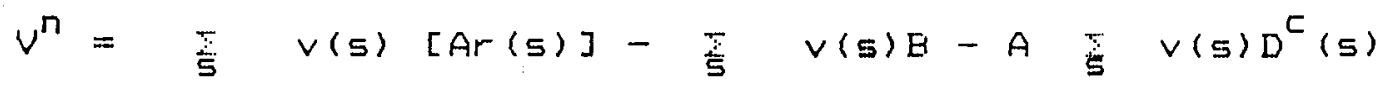

$$
\begin{aligned}
& =A\left[\underset{5}{5} \vee(5) r(s)-\frac{F}{5} v(5) D^{C}(5)\right]-B \quad v(5)
\end{aligned}
$$

Note that:

$$
\begin{aligned}
& \text { at } x=0, \quad \frac{5}{5} \quad v(5) r(5)=\frac{5}{5} \quad v(s) D^{1}(5) \\
& \text { at } x=1, \quad v(5) r(5)=\quad \forall(5) D^{2}(5)
\end{aligned}
$$


Given the tax effects. the expression in brackets is clearly maximized at $x=0$. Woreover, at $x=0$ this expression is positives. Conditional on the choice of an optimal $x$ value, $V^{n} i s$ thus maximized at $A=A_{\text {max }}$.

This is the conclusion reached by Elact: and Tepper. Dur setting is different -.- we rely on a marlet structure, while they use arbitrage arguments that require explicit offsetting actions, but the conclusions are the same.

The Blact-Tepper result involves the use of pension funding and investment in bonds to take advantage of an asymetric tas structure. Folicjes that involve possible default by the fund provide a way to take advantage of possible asymetric behavior on the part of the FBGC, the insurer of pension benefits. If stocks are more effective than bonds in this role, and if the feasible $(A, X)$ region includes combinations that make default possible, the Elack-Tepper strategy may not maximize shareholder wealth.

The matter is not straightforward, even in this very simple setting. The "value of the insurance" depends on the relationship between the payoffs over states of (1) fund assets and (2) benefits. It is entirely possible that a risky bond might provide both tax benefits and a large value for the insurance. In effect, we are assuming that the manager will consider both the "tax effect" and the "insurance offect" of decisions concerning funding and investment. Our previous results indicate that in our setting, the optimal policy will involve an extreme point in the feasible $(A, X)$ space, whether the choice of "bonds" versus "storks" involves a trade-off of these two effects or rot. It is instructive, however, to examine a very simple case in which there is such a tradeoff.

Assume that the payoffs from the two instruments are

$$
\text { Bonds: } \quad \begin{aligned}
D^{1}(1) & =1.1 \\
D^{1}(2) & =1.1
\end{aligned}
$$

Stocks: $\quad D^{2}(1)=1.2$ 


$$
0^{2}(2)=.1
$$

The valuation function is

$$
\begin{aligned}
& v(1)=.85 . \\
& v(2)=.10 .
\end{aligned}
$$

We assume that

$$
\frac{1}{5} \quad v(s) D^{C}(s)=1
$$

Note that there is a "tax effect", since

$$
\begin{aligned}
& \frac{5}{5} \quad v(5) D^{1}(5)=1.045, \\
& \frac{5}{5} \quad v(5) D^{2}(5)=1.030, \\
& \frac{6}{5} \quad v(5) D^{C}(5)=1.000 .
\end{aligned}
$$

Eenefits are 110, regardless of state, and regulatory constraints 5 are

$$
\begin{aligned}
& A_{\text {max }}=120, \\
& A_{\text {min }}=100, \\
& x_{\text {max }}=1.0 . \\
& x_{\text {min }}=0.0 .
\end{aligned}
$$

Note that investment solely in bonds will cover benefit payments in every state, even at the minimum feasible funding level. Thus the value of the insurance will increase with $x$.

Figure 4 shows the feasible region and the value of $v^{n}$ at each corner las indicated earlier, the absolute value can be interpreted as the cost of the pension plan). 
One might think that, given the tradeoff between the insurance and tax effects, and our previous results, the optimal policy would involve either (a) full funding plus investment in bonds (corner 1 in Figure 4) or (b) minimum funding plus investment in stoctss (corner $\Xi$ in Figure 4). However, as our example shows, this need not be the case. Here the cost of the plan is minimized by a policy involving full funding and investment solely in stocks (corner 2 ). 


\section{Suminary and Conclusions}

The main conclusion of this paper is the indication that a fund manager should focus on extreme funding and investment strategies if oum assumptions about regulatory constraints are valio. The Ifis and the FEGC, on the other hand, may want to adopt regulatory policies such that fund managers' optimal strategies will be more consigtent with broader social objectives. 


\section{FOOTNOTES}

* The comments and suggestions of Jeremy Eulow. Myron Scholes and Fiobert Litzenberger are gratefully aclinowledged.

1. Tepper makes this assumption explicitly, Blacts implicitly.

2. since the only source of uncertainty is mortality, appeal may be made to the law of large numbers for justification.

S. This rules out decisions made to maximize the utility of ristu-averse managers or shareholders who are unable to diversify sufficiently to regard the decision as one of maximizing current weal th.

4. realistically, given ERISA and the behavior of the FBGC

5. Given the contingent liability of the corporate sponsor under EFISA, the values of $A$ should include $30 \%$ of the corporation"s net worth. With this interpretation, our numeric example is not overly fanciful. 


\section{REFEFENNCES}

1. Flack, F. 1980. The Tax Consequences of Long-run Fension Folicy. Financial Analyst." Journal 36,4: 21-28.

2. Litzenberger, Fi.H. and Van Horne J. 1979. Elimination of The Double Taxation of Dividends and Corporate Financial Folicy.

Journal of Finance ES: 757-749.

3. Schaefer, 5. 1978. Taxes and Security Market Equilibrium. Stanford University Graduate School of Fusiness Fesearch Faper No. 568.

4. Sharpe,W.F. 1976. Corporate Fension Funding Folicy. Journal of Financial Economics 3: 18J-194.

5. Tepper, I. 1981. Taxation and Corporate Fension Foljey. Journal of Finance $36,1: 1-13$.

6. Tepper, I. 1982. The Future of Frivate Fension Funding. Financial Analyst. Journal $38,1: 25-31$. 


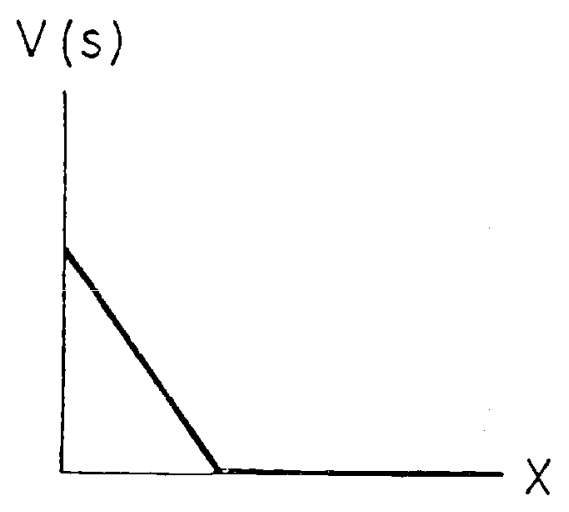

(a)

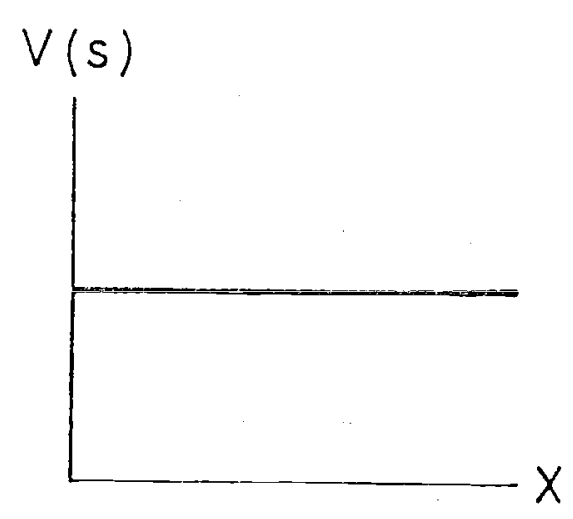

(b)

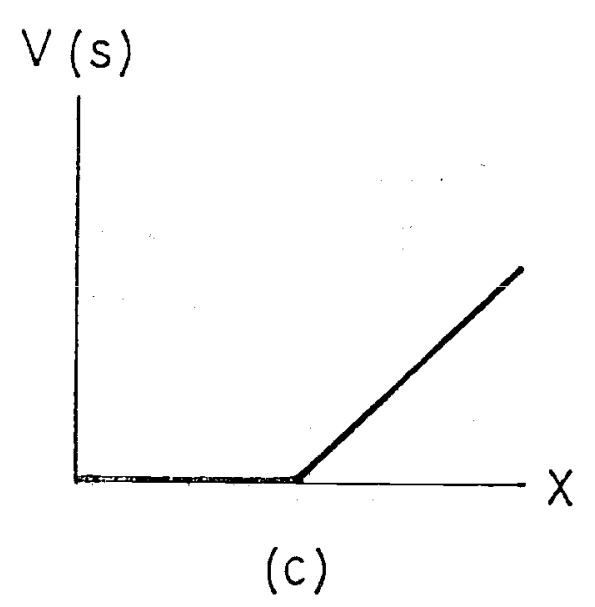

FIGUFE 1. Convexity of $V(s)$ as a Function of $x$ 


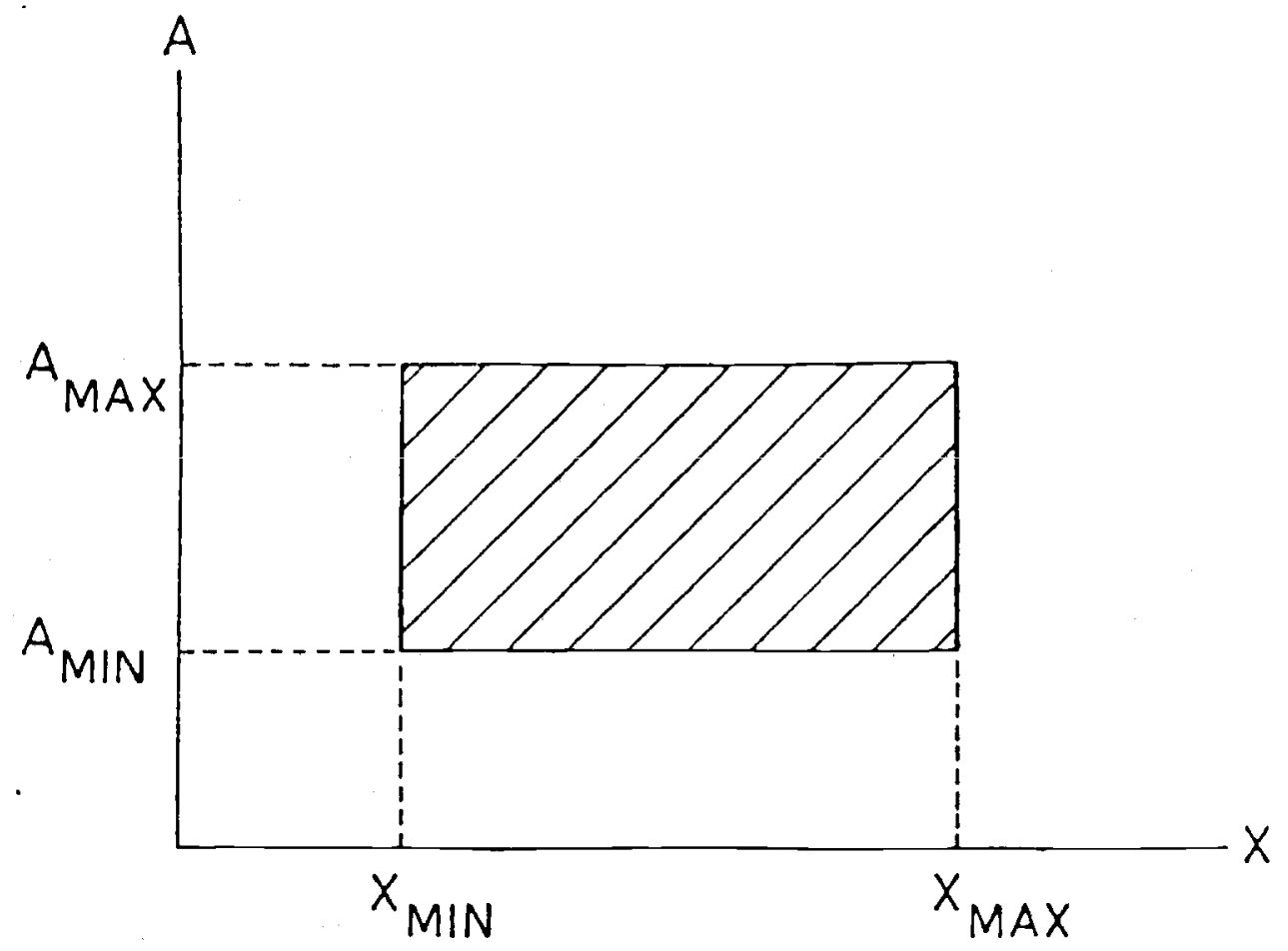

FIGUFE 2. The Feasible Fiegion with simple Fiegulatory Constraint 


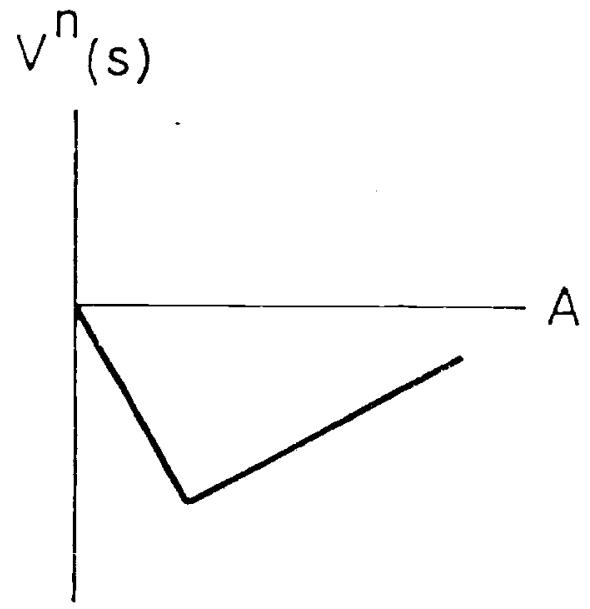

(a) $v^{n}(s)$

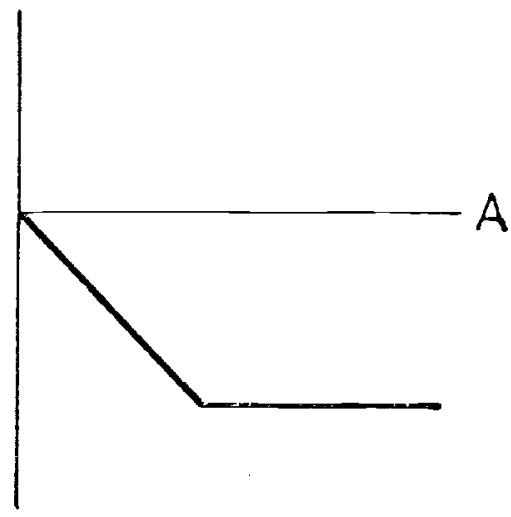

(b) $v^{n}(s)$

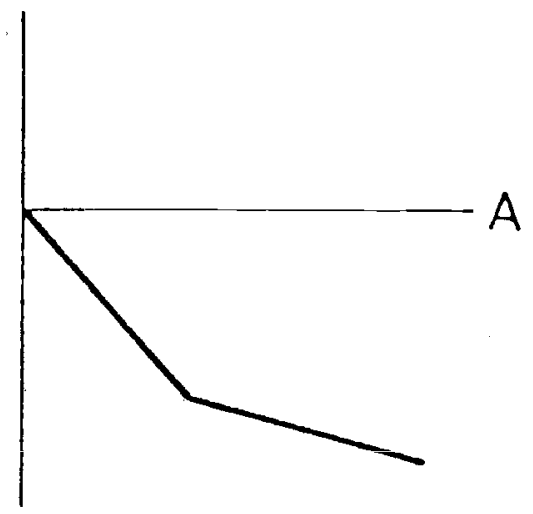

(c)

FIGUFE 3. Convexity of $V^{n}(s)$ as a Function of $A$ 


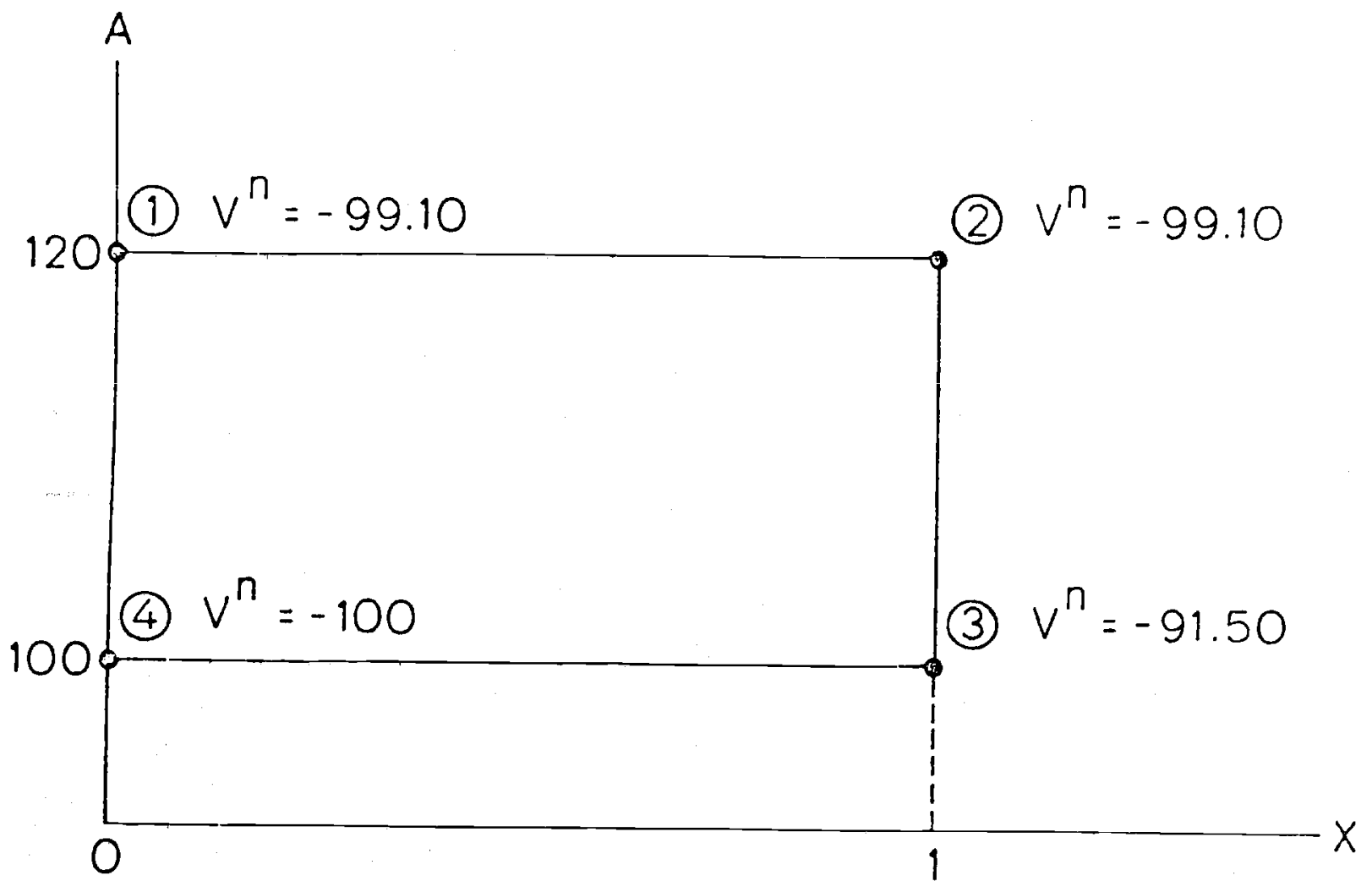

FIGUFE 4. Values of $v^{n}$ at the Four corners of the Feasible fiegion 